\title{
Realistic Magnetospheric Mass Density Model For 29 August 2000
}

\author{
R.E. Denton ${ }^{a}$ R.E. Denton J. Goldstein ${ }^{b}$ D.-H. Lee ${ }^{c}$ \\ R.A. King ${ }^{d}$ Z.C. Dent ${ }^{e}$ D. Berube ${ }^{f}$ D.L. Gallagher ${ }^{\mathrm{g}}$ \\ K. Takahashi ${ }^{\mathrm{h}}$ M. Nose ${ }^{\mathrm{i}}$ D. Milling ${ }^{\mathrm{e}}$ A. Keiling ${ }^{\mathrm{j}}$ F. Honary ${ }^{\mathrm{k}}$ \\ ${ }^{a}$ Dartmouth College, Department of Physics and Astronomy, 6127 Wilder Lab, \\ Hanover, NH 03755, USA \\ ${ }^{\mathrm{b}}$ Southwest Research Institute, Space Science and Engineering Division (15), 6220 \\ Culebra Rd., San Antonio, TX 78238, USA \\ ${ }^{\mathrm{c}}$ Kyung Hee University, Department of Astronomy and Space Science, Yongin, \\ Kyunggi-Do, 449-701, Republic of South Korea \\ ${ }^{\mathrm{d}}$ University of Arizona, 901 GS LPL West, 1040 E 4th St., Tucson, AZ 85721, \\ USA \\ e University of Alberta, Space Physics Group, Department of Physics, Edmonton, \\ Alb T6G 2J1, Canada \\ ${ }^{\mathrm{f}}$ UCLA, 595 Charles Young Dr East, 3806 Geology Bldg., Los Angeles, CA \\ 90095-1567, USA \\ ${ }^{\mathrm{g}}$ NASA Marshall Space Flight Center, 1321 Shadow Ridge Rd., Huntsville, AL \\ 35803, USA \\ h Johns Hopkins University, Applied Physics Lab, 11100 Johns Hopkins Rd., \\ Laurel, MD 20723-6099, USA \\ ${ }^{\mathrm{i}}$ Kyoto University, Data Analysis Ctr. Geomagnetism 83 Space, Oiwake-cho \\ Kitashirakawa Sakyo-ku, Kyoto, 606-8502, Japan \\ ${ }^{\mathrm{j}}$ CESR, 9 avenue du Colonel Roche, BP 4346, Toulouse Cedex 4, 31028, France \\ ${ }^{\mathrm{k}}$ University of Lancaster, Department of Communication Systems, Lancaster LA1 \\ $4 Y R$, Great Britain
}

\begin{abstract}
Using a two dimensional image of the Earth's plasmasphere taken by the Extreme Ultraviolet Imager (EUV) on the Imager for Magnetopause-to-Aurora Global Exploration (IMAGE) spacecraft, in-situ electron density measurements from the IMAGE Radio Plasma Imager (RPI) instrument, measurements of magnetospheric mass density inferred from field line resonant frequencies measured by magnetometers on the Earth's surface, and a model for the density field aligned variation, we construct a computer model for the magnetospheric mass density on 29 August 2000 at 1519
\end{abstract}


UT. Such a model can be useful for studies of magnetospheric phenomena such as ultra low frequency Pi-2 oscillations and plasmaspheric cavity modes, and for calibrating IMAGE EUV density inversion schemes. This study also sheds new light on the distribution of magnetospheric density through its detailed evaluation of the density in various regions.

Key words:

plasmasphere, density model, mass density, electron density, EUV images

PACS: 94.30.Fk, 07.05.Tp, 07.05.Kf, 52.70.Kz, 52.70.6w, 53.35.Bj

\section{Introduction}

The plasmasphere is the region of large plasma density surrounding the Earth (Wolf et al., 1995). It typically extends several Earth radii out from the Earth's surface along the magnetic equator, and the plasmaspheric density extends along the Earth's dipole field lines toward the magnetic poles. Recently, the Extreme Ultraviolet Imager (EUV) on the Imager for Magnetopause-toAurora Global Exploration (IMAGE) spacecraft has been able to image the ultraviolet emission of plasmaspheric helium (Sandel et al., 2001). Ideally, one would hope to use these images to extract a map of magnetospheric density. So far, such a task has been difficult. Difficulties relate to the relation of emission to local density, the line of sight integration of the emission, lack of emission at low density, and uncertainties about the field line distribution of density and the relative density of various plasma species. Here, we generate a computer model for the magnetospheric electron density and mass density at 29 August 2000, 1519 UT, by supplementing the EUV data with in-situ electron density measurements at earlier and later times (Bauer and Stone, 1968; Benson et al., 2004), and with mass density values inferred from field line resonance frequencies measured by magnetometers on the Earth's surface (Menk et al., 1999).

The mass density controls the rate of response of the magnetosphere to internal or external perturbations. The purpose of this particular study is to derive a realistic mass density model to study the propagation of fast/magnetosonic magnetohydrodynamic waves through the plasmasphere and the resonance properties of these waves within the plasmaspheric cavity. (The waves may resonate within the plasmasphere much like sound waves resonate in a musical instrument.) A previous study using a two dimensional model of the plasmasphere found that the plasmaspheric cavity resonance could account

Email address: richard.e.denton@dartmouth.edu (R.E. Denton). 


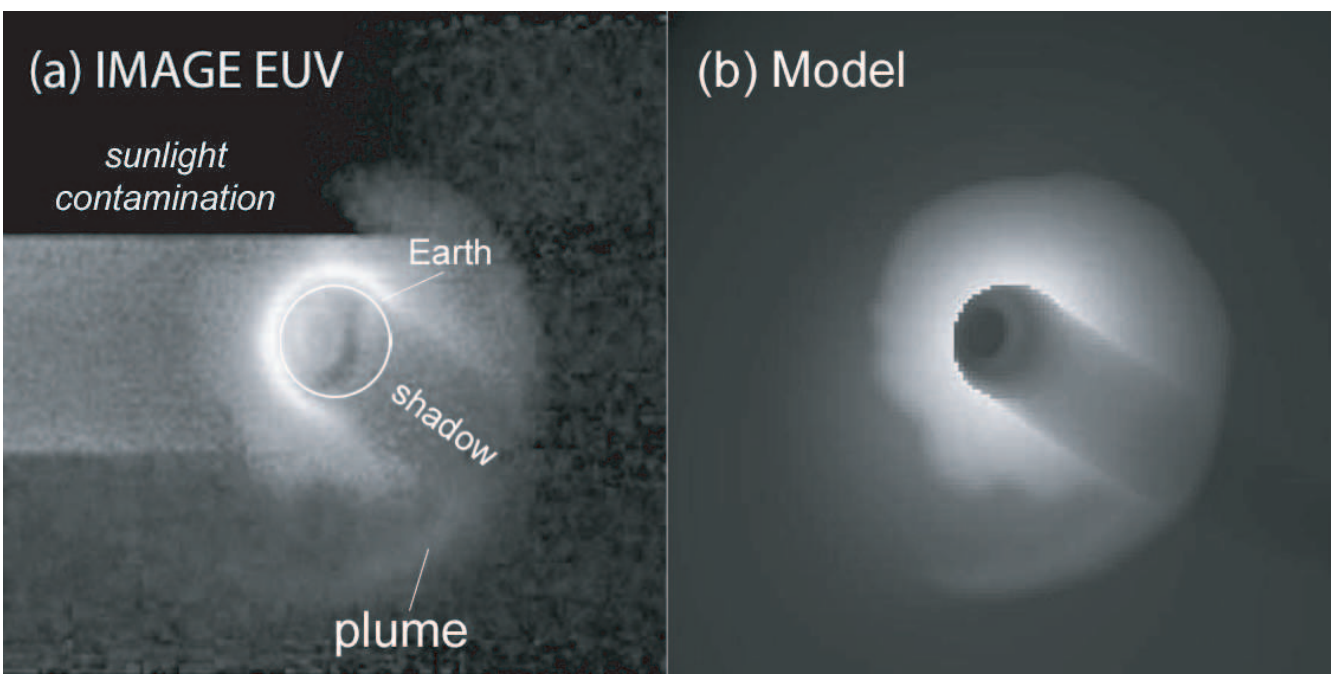

Fig. 1. (a) EUV instrument image of resonantly scattered solar EUV photons (30.4-nm) at 29 August 20001519 UT. (b) Simulated EUV instrument image using the model for electron density developed in this paper.

for the observed ultra low frequency (ULF) frequencies of Pi-2 oscillations of the Earth's magnetic field (Denton et al., 2002). However, this study did not address the effects of a realistic three dimensional density model on the waves. The density model described in this paper has been derived for the purpose of investigating these waves, but such a model can be used in many other dynamical studies. The model could also be useful for calibrating IMAGE EUV density inversion schemes. Furthermore, this study also sheds new light on the distribution of magnetospheric density in certain regions, such as in the dusk plasmatrough and plume.

\section{Development of the Model}

\subsection{EUV Image}

The IMAGE EUV instrument images solar EUV photons at 30.4-nm wavelength that are resonantly scattered by singly ionized helium in the plasmasphere (Sandel et al., 2001). The 30.4-nm feature is relatively easy to measure because it is the brightest ion emission from the plasmasphere, it is spectrally isolated, and the background at that wavelength is negligible. The plasmaspheric He+ emission is optically thin, so its brightness is directly proportional to the He+ column abundance. Full images are taken with at least $10 \mathrm{~min}$ resolution.

Figure 1a shows the plasmaspheric image taken at 29 August 20001519 UT. The Sun is off the plot in the direction of the upper left corner; on the lower 
right side of the plot there is darker emission due to the Earth's shadow. The image is a composite from three cameras; this is the cause of the horizontal discontinuities in brightness. There is significant sunlight contamination on the left side of the plot, particularly for the middle camera. Much of the field of view of the upper camera is shielded to avoid damage to the camera. The increasing brightness toward the center of the image corresponds to higher density in the plasmasphere. The brightest (horseshoe shaped) ring comes from emission close to the surface of the Earth. (This emission is actually a combination of $\mathrm{He}+$ emission other than 30.4-nm and $\mathrm{O}+$ emission that is so bright that it is detected by EUV (Bill Sandel, private communication, 2004).) Considerable structure in the shape of the plasmasphere is evident; note particularly the plasma plume (or tail) at the bottom of the plot and curving around toward the left side of the plot.

\subsection{Plasmapause Position}

The plasmapause is the outer boundary of the high density plasmasphere. At the plasmapause, the density falls off steeply. The region outside the plasmasphere is called the plasmatrough. In Figure 1a, there is an irregular boundary where the 30.4-nm emission falls off sharply. Goldstein et al. (2003) calls this the "He+ edge", and shows that this visual feature corresponds to the location of the plasmapause (sudden drop in electron density). This drop in bright emission may and often does cross the EUV instrument threshold $\sim 40 \pm 10 \mathrm{~cm}^{-3}$.

The asterisks in Figure 2 show the plasmapause determined using the method of Goldstein et al. (2003). The image of Figure 1a has now been rotated into Solar Magnetic (SM) coordinates, with $X$ in the direction of the Sun (toward the left of the plot), and $Y$ in an eastward direction (toward the bottom of the plot). Thus the Sun is to the left in Figure 2. The half illuminated circle at the center of the plot shows the location of the Earth. The location of the plasmapause at local noon (facing the Sun) is not indicated by asterisks in Figure 2 because the corresponding region of the image in Figure 1a is blacked out to protect the camera.

The solid curve in Figure 2 shows the location of the plasmapause used in the model. The plasmasphere rotates with the Earth in a counterclockwise direction (viewing the Earth from the north). At dawn local time (top side of Figure 2 ), the rotation of the plasmasphere is usually close to but somewhat faster than the Earth's rotation, while at dusk (the bottom side of Figure 2), the rotation is close to but somewhat slower (Wolf et al., 1995; Burch et al., 2004). Thus by looking at the plasmapause at dawn in earlier EUV instrument images or at dusk in later ones, we can get an idea of the plasmapause structure at 1519 UT (time corresponding to Figure 1a) at local noon. Using EUV 


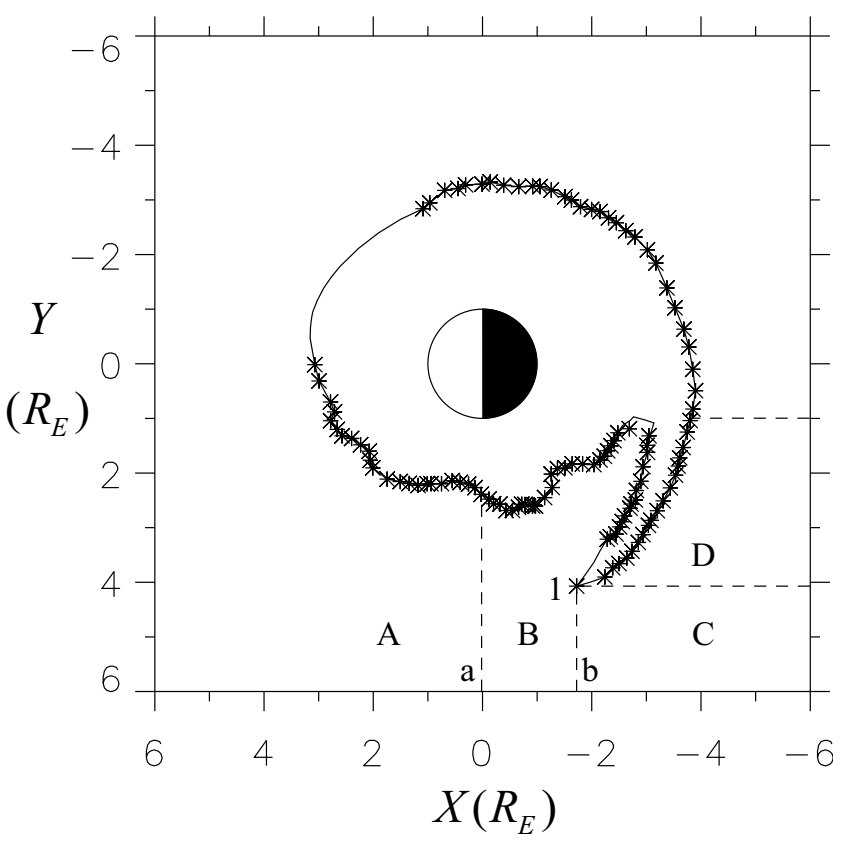

Fig. 2. Plasmapause position in SM coordinates $X$ and $Y$ in units of Earth radii $R_{E}$. The asterisks show the plasmapause determined using the method of Goldstein et al. (2003). The solid curve shows the plasmapause used in the model. The regions $\mathrm{A}, \mathrm{B}, \mathrm{C}$, and D, lines a and b, and point 1 are discussed in Section 2.5.

images before and after 1519 UT, no large amount of structure was observed for this region, so we connected the measured plasmapause positions at early morning local time to that in at early afternoon local time with a roughly radial connection. A slight bulge in the plasmapause was included at magnetic local time MLT $\sim 10.7$ to bring the plasmapause out to $L=3.2$ at that local time (see discussion in Section 2.5). A smooth connection of the asterisks was also made in the plasma plume.

\subsection{Electron Density in the Inner Plasmasphere}

The IMAGE spacecraft has a meridional orbit (orbit over the poles). In 2000, its apogee was over the North pole, and that is where the image in Figure 1a was taken. After 1519 UT, the spacecraft moved toward MLT $\sim 2.5$ (postmidnight) as it approached perigee at a height of $1.2 R_{E}$. It then passed over the South pole and moved outward at MLT $\sim 13.7$ (afternoon). Because of this, IMAGE passed through the plasmasphere at two MLT values within 8-11 hours UT after 1519 UT. Similarly, IMAGE passed through the plasmasphere at two different MLT values within 3-6 hours UT before 1519 UT.

The Radio Plasma Imager (RPI) instrument on IMAGE (Reinisch et al., 2000), when operated in its passive mode, measures emission which is associated with the upper hybrid frequency, and the frequency of this emission can be used 


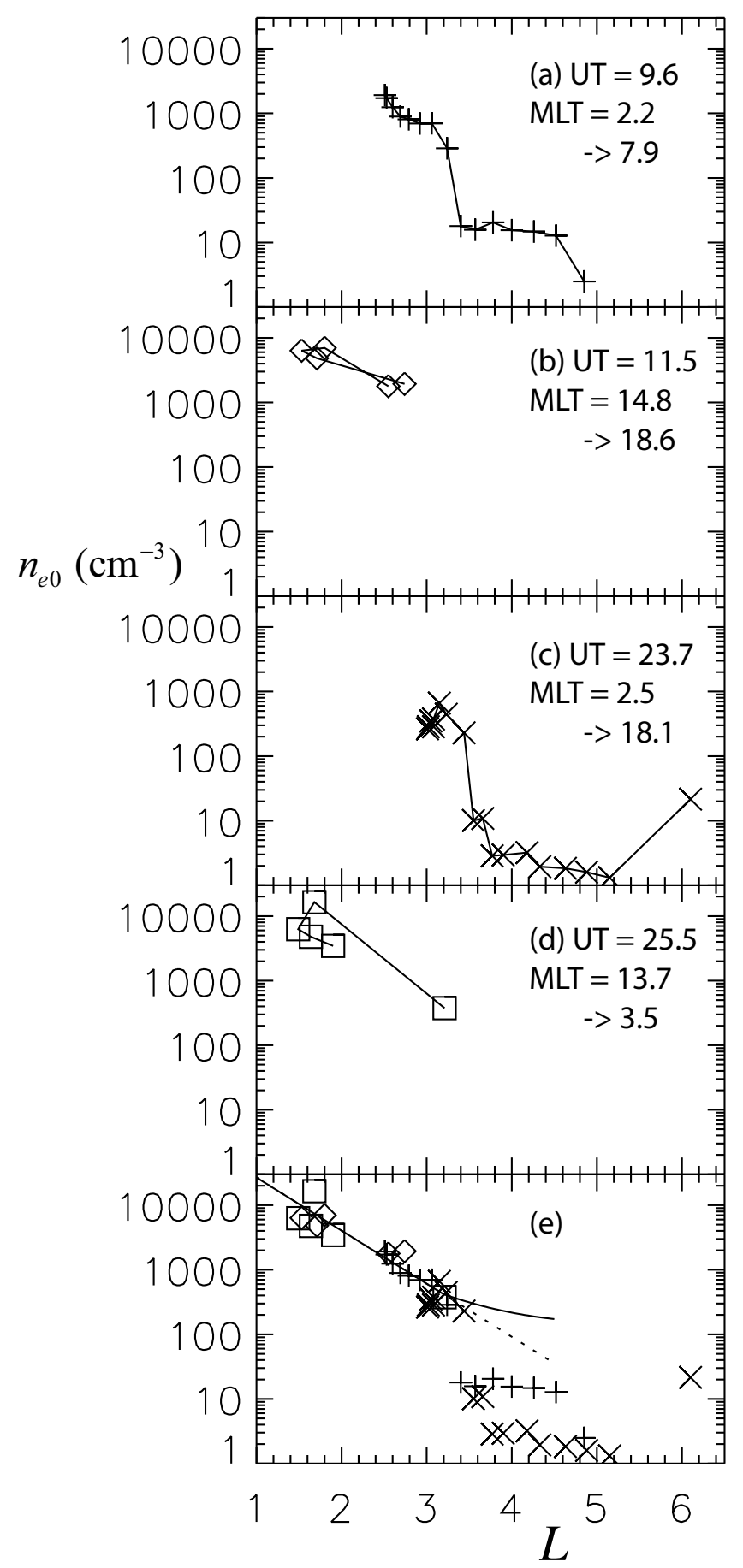

Fig. 3. (a-d) In-situ electron density $n_{e}$ versus $L$ as measured by IMAGE RPI at universal times close to the values indicated in each panel. The two MLT values given in each panel in the format $\mathrm{MLT}_{1} \rightarrow \mathrm{MLT}_{2}$ are the MLT value where $n_{e}$ was measured $\left(\mathrm{MLT}_{1}\right)$ and the mapped value of MLT assuming corotation $\left(\mathrm{MLT}_{2}\right)$. (e) All the measurements superposed. 
to infer the local electron density $n_{e}$ (Bauer and Stone, 1968; Benson et al., 2004). These measurements of the local electron density determined before and after 1519 UT are shown in Figure 3a-d for the four UT and MLT values indicated in each panel. (Both the UT and MLT values are typical values approximating a range of values. Figures $3 \mathrm{~b}$ and $\mathrm{d}$ plot the density values for consecutive time values near spacecraft perigee where $L$ first decreases, then increases; that is why the $L$ values are not monotonic.) The MLT values are shown in the format $M_{1} \rightarrow \mathrm{MLT}_{2}$. The first value $\mathrm{MLT}_{1}$ is the local MLT where the measurements were taken, and $\mathrm{MLT}_{2}$ is the mapped value of MLT assuming corotation. For instance, the measurements of $n_{e}$ in Figure 3b were measured at $11.5 \mathrm{UT}$ at MLT $=14.8$. This is 3.8 hours UT earlier than 1519 $=15.3$ UT. Assuming then that the plasma observed in Figure 3b corotated with the Earth from 11.5 to 15.3 (1519) UT, it would have moved from MLT $=\mathrm{MLT}_{1}=14.8$ to MLT $=\mathrm{MLT}_{2}=14.8+3.8=18.6$ by 1519 UT.

The data at all four times is superposed in Figure 3e. While there is a significant variation in the low density values with $n_{e}<40 \mathrm{~cm}^{-3}$ (plasmatrough), the high density values (plasmasphere) fit fairly well on the dashed line

$$
\log _{10}\left(n_{e}\right)=5.25-0.82 L
$$

With a slight modification (described in the Section 2.4), we use Equation 1 to describe $n_{e}$ within $L=3.2$.

\subsection{MLT Dependence in the Inner Plasmasphere}

A code has been developed to invert the EUV instrument image and get a "pseudo-density" of $\mathrm{He}+, n_{\mathrm{He}}$. Counts are first translated into integrated column density using the SOLAR2000 solar flux model (Tobiska, 2000). The assumption is made that the plasmaspheric densities drop rapidly with L-shell and that the dominant contribution to image intensity in any single pixel comes from that portion of the line of sight that passes within $0.1 \mathrm{~L}$ of the minimum L-shell. The length of that segment in the line of sight increases with increasing L-shell for an observing location at high latitude and can result in a bias toward larger L-shell of as much as a factor of three. Column density in $\mathrm{cm}^{-2}$ is converted to density in $\mathrm{cm}^{-3}$ by dividing by the length of that segment of the line of sight.

Density structure is fairly well ordered by the Earth's magnetic field, which is approximately dipolar. In three dimensions, the density structures evident in the equatorial plane (structures similar to, but not exactly the same as those shown in Figure 1a) drape up out of the equatorial plane along magnetic field lines and turn down toward the poles. The calculation of pseudo-density 


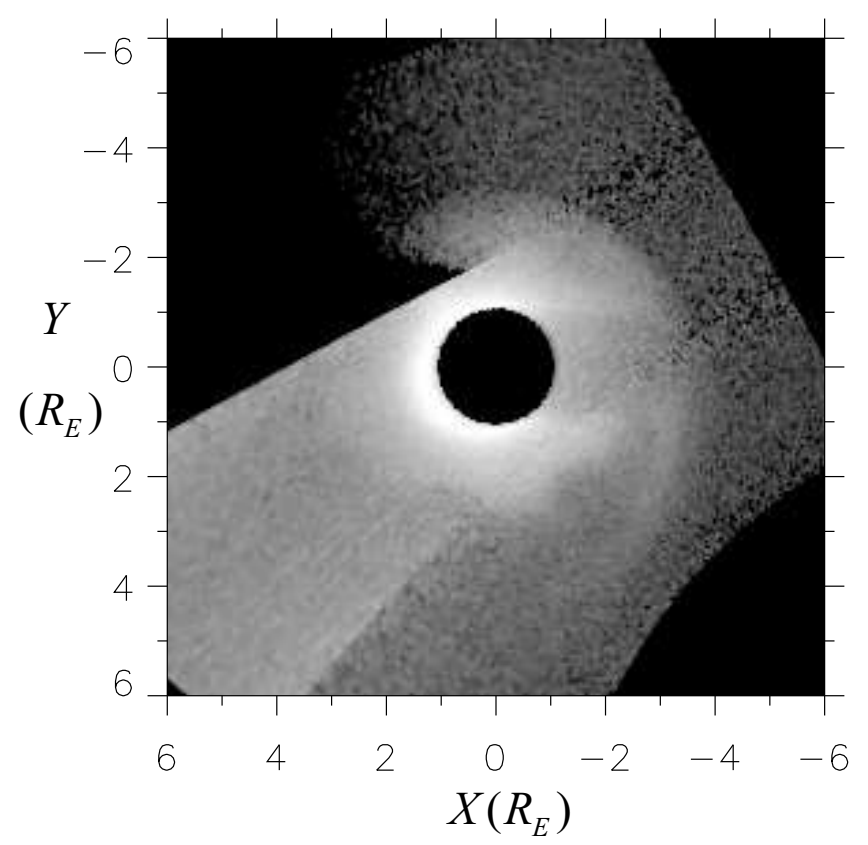

Fig. 4. Grayscale plot of the $\log$ of the pseudo-density of $\mathrm{He}+, n_{H e}$, versus SM coordinates $X$ and $Y$ using Figure 1a as an input.

does not take into account overdraped field line content (high density at large $L$ that because of the shape of the magnetic field drapes across the field of view looking toward a lower $L$ in the equatorial plane). That means that low pseudo-densities radially inward of a density enhancement (especially the plume) would be expected to be somewhat higher than the actual density.

Figure 4 shows a greyscale plot of the log of the He+ pseudo-density $n_{H e}$ using Figure $1 \mathrm{a}$ as an input. Figure 5 shows the pseudo-density $n_{H e}$ versus $L$ at four different values of MLT, MLT $=18$ (thin solid curve), 21 (thick solid curve), 3 (small dashed curve), and 6 (large dashed curve). From the pseudo-density, we can see several features of the plasmasphere, a steeply dropping density within the plasmasphere proper (i.e., within $L \leq 3$ ), a drop to a much lower density at large $L$ (except for MLT $=18$ ), and the plasma plume (bump in the thick solid curve at $L \sim 3.8$ ).

Ideally, we would just use the density plotted in Figure 4 and Figure 5 and multiply by the electron to He+ ion ratio to get the electron density. However, the uncertainties in the method leading to Figure 4 are too great to follow that procedure. If we assume a constant $n_{e} / n_{H e}$ ratio, the densities in Figure 4 are not consistent with the data presented elsewhere. If we use the Craven et al. (1997) model for $n_{e} / n_{H e}$, the disagreement is worse. (The data of Craven et al. (1997) has $n_{e} / n_{H e}$ increasing with respect to $L$. The pseudo-density slightly decreasing with respect to $L$ in the plasmatrough at most values of MLT in Figure 5 would imply that $n_{e}$ is increasing with respect to $L$, contrary, for the most part, to the profiles for $n_{e}$ in Figure 3.) Because of this, we use a 


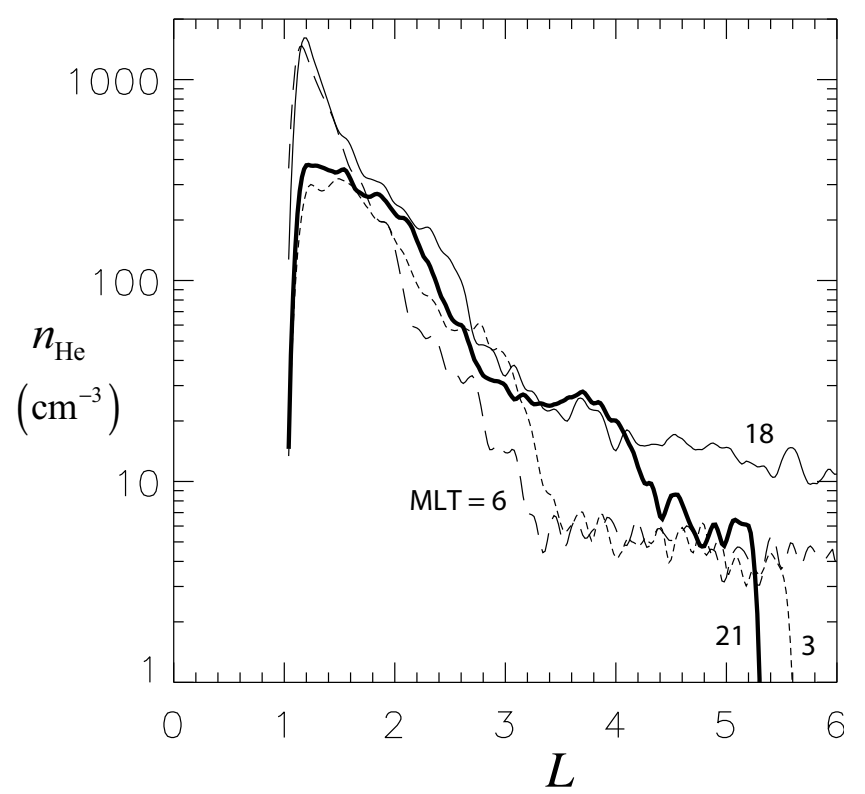

Fig. 5. Pseudo-density of $\mathrm{He}+, n_{\mathrm{He}}$, versus $L$ along four cuts, MLT $=18$ (thin solid), 21 (thick solid), 3 (small dashes), and 6 (large dashes).

constant $n_{e} / n_{\mathrm{He}}$ ratio in our model and we use the pseudo-density as a guide for some limited aspects of the model.

First we note that the density in the inner plasmasphere $(L \leq 3.2)$ has a small MLT dependence. The solid curves in Figure 5, representing density on the duskside, are somewhat higher than the dashed curves, representing the density on the dawn side. We incorporate this into the model by modifying Equation 1 as follows:

$$
\log _{10}\left(n_{e}\right)=5.25-0.82 L+0.25(L-1.5) \cos (2 \pi(M L T-18) / 24),
$$

where MLT is given in hours. The second observation is that the density in the plume (peak in the thick solid curve at $L=3.8$ in Figure 5) is larger than one would expect based on the dropoff in density up to about $L=3$ (thick solid curve at $L \leq 3$ ). Because of this, our plasmasphere density decreases less strongly outside $L=3.2$ (solid curve in Figure 3e). Further motivation for the choice of the outer $(L>3.2)$ plasmaspheric density is presented in Sections 2.5 and 3. Also, because of the problems understanding the $n_{e} / n_{H e}$ ratio mentioned above (in this subsection), we assume that the average ion mass (ratio of mass density to electron density) is constant throughout the magnetosphere. 


\subsection{Mass Density Based on Field Line Resonance Frequencies}

The field line resonance mode is an azimuthally oscillating (toroidal) Alfven wave. The resonance frequency is particularly dependent on the mass density in the vicinity of the magnetic equator, where the magnetic field strength is a minimum (Denton and Gallagher, 2000). Given a realistic magnetic field model, the magnetospheric mass density in the vicinity of the magnetic equator can be determined from field line resonance frequencies measured by magnetometers on the Earth's surface. Here we use data from the SAMNET (SubAuroral Magnetometer Network, http://www.dcs.lancs.ac.uk/iono/samnet/, Yeoman et al., 1990), IMAGE (International Monitor for Auroral Geomagnetic Effects, http://sumppu.fmi.fi/image/, Luhr et al., 1998), BGS (British Geological Survey, data available from SAMNET) ground magnetometer arrays in Europe and Antarctica, and from the MEASURE (Magnetometers Along the Eastern Seaboard for Undergraduate Research, http://measure.igpp.ucla.edu/, Berube et al., 2003) ground magnetometer array. The resonant field line was determined using the cross phase technique with pairs of stations (Waters et al., 1991)). For each measurement, the T96 magnetic field model (Tsyganenko, $1995)$ is used to map the ground station location to the equatorial plane where the magnetic field is a minimum. The normalized frequency of the Alfven wave fundamental $\bar{f}_{1} \equiv f_{1} L R_{E} / V_{A-\text { Bmin }}$ is calculated using the dipole field model (as described by Denton and Gallagher (2000)), but for $L R_{E}=R_{\mathrm{Bmin}}$, where $R_{\mathrm{Bmin}}$ is the geocentric radius to the point on the field line where $B$ is a minimum according to the T96 model, and $V_{A-B m i n}$ is the Alfven speed at that location. A perfect conductor boundary is assumed at a height of $400 \mathrm{~km}$ above the ground, and $\rho$ is assumed to vary along field lines like $R^{-0.5}$, where $R$ is the geocentric radius. (The normalized frequency is not a strong function of $L$ except at very low $L \leq 2$; except for the point with $R_{\mathrm{Bmin}}=1.9 R_{E}$, for which $\bar{f}_{1}=0.42, \bar{f}_{1}$ is equal to $0.35 \pm 0.02$ for all our data points.) Then the mass density at the magnetic equator is found from

$$
\rho=\left(3.42 B_{\min } \bar{f}_{1} /\left(G R_{\mathrm{Bmin}}\right)\right)^{2},
$$

where $B_{\min }$ and $R_{\mathrm{Bmin}}$ are found from the T96 model, and $G$ is the ratio of the total length of the T96 field line to that of a dipole field line with $L R_{E}=R_{\text {Bmin }}$ ( $G$ is within $8 \%$ of unity for all our data points). Using the values of $B_{\min }$ and $R_{\text {Bmin }}$ from the T96 magnetic field in this way results in a more accurate estimate of $\rho$ than would be found from the dipole field model alone.

Figure 6a shows mass density values inferred from field line resonance mode frequencies at dusk local time, MLT $\sim 17.6$ (asterisks, Scandinavian meridian) and 15.8 (diamonds, UK meridian). Figure 6b shows two inferred mass density values at morning local time, MLT $\sim 10.3$ (asterisks, MEASURE). Unlike the data shown in Figure 3, these density values are based on measurements at UT 


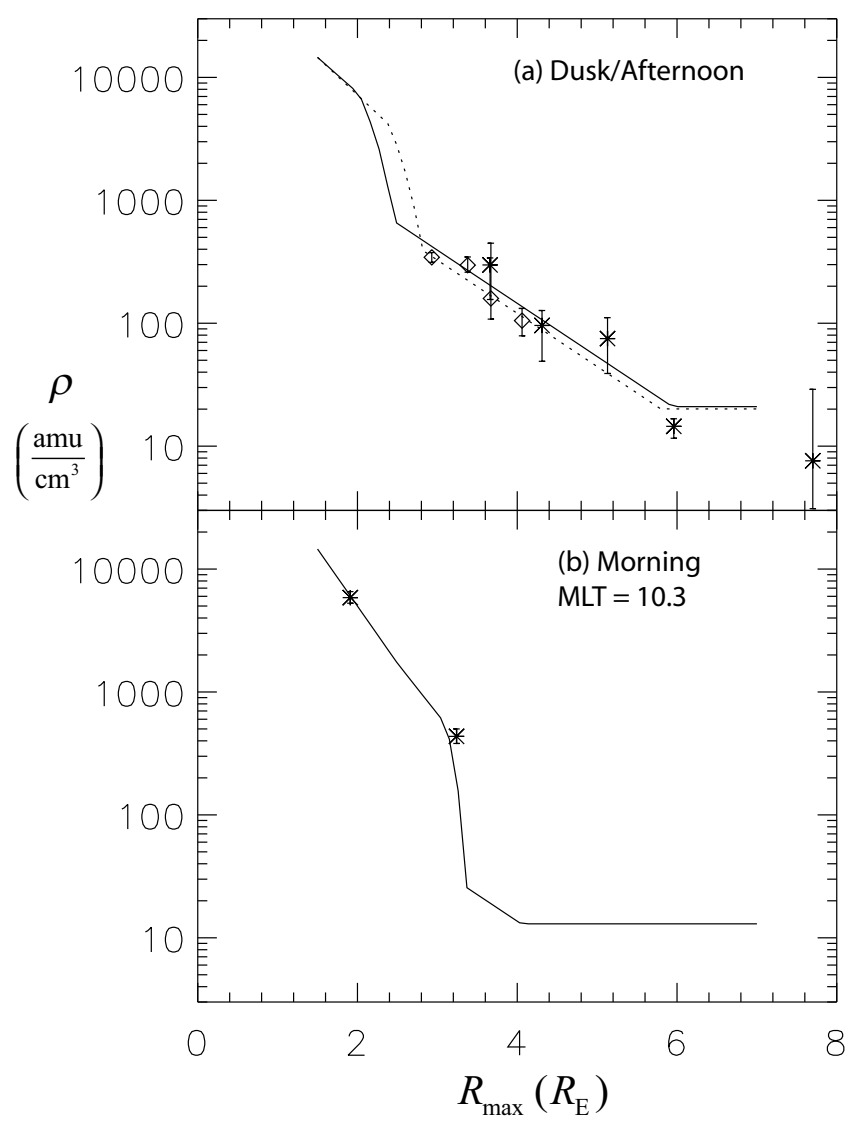

Fig. 6. (a) Inferred mass density at UT 1519 (time of EUV image in Figure 1a) in the vicinity of the magnetic equator versus $L$ based on field line resonance frequencies measured by Scandinavian meridian ground magnetometers (asterisks) at MLT $\sim 17.6$ and by UK meridian ground magnetometers (diamonds) at MLT 15.8, and our model mass density at MLT $=17.6$ (solid curve) and at MLT $=$ 15.8 (dashed curve). (b) Inferred mass density based on frequencies measured by MEASURE ground magnetometers (asterisks) at MLT $\sim 10.3$, and our model mass density at MLT $=10.3$ ( solid curve).

$\sim 1519$ (time of EUV image in Figure 1a). The curves in the plots represent the mass density from our model, where we have assumed an average ion mass of $1.4 \mathrm{amu}$ throughout the magnetosphere. This factor gives rough agreement between the plasmaspheric mass density values at dawn (Figure 6b) and the RPI local electron density values (Section 2.3), as indicated by the agreement of the asterisks and solid curve in Figure 6b.

Based on the mass density values in Figure 6a, we include in our model a region of enhanced density concentrated in the dusk local time sector and outside the plasmasphere proper (outside $L \sim 2.5$ at MLT $=18$ ). Based on the radial dependence in Figure 6a, we model this density as

$$
\log _{10}\left(n_{e}\right)=3.75-0.43 L
$$


As can be seen from Figure 6a, the density in this region falls off less steeply with respect to $L$ than does the density in the plasmasphere at $L \leq 2.4$. Equation 4 describes the density in region $\mathrm{B}$ of Figure 2. This density component also contributes to the density in regions A, C, and D of Figure 2; however, in these regions the density in Equation 4 is multiplied by a factor that decreases with respect to the distance away from region B. As can be seen from Figure 1, the EUV emission from the region outside the plasmasphere in region $\mathrm{B}$ falls off in the other regions, particularly toward midnight local time. We chose a $1 R_{E}$ spatial scale for the falloff of the density toward midnight, and a $5 R_{E}$ spatial scale for the falloff of the density toward noon. In region $\mathrm{A}$, the density in Equation 4 is multiplied by $\exp \left(-((18-M L T) / 5)^{2}\right)$; in region $\mathrm{C}$, the density in Equation 4 is multiplied by $\exp \left(-\left(X_{1}-X\right)^{2}\right)$, where $X_{1}$ is the $X$ coordinate of point 1 in Figure 2; and in region $\mathrm{D}$, the density in Equation 4 is multiplied by $\exp \left(-\left(\left(X_{1}-X\right)^{2}+\left(Y_{1}-Y\right)^{2}\right)\right)$, where $Y_{1}$ is the $Y$ coordinate of point 1 in Figure 2.

For the outer measurement at $L \sim 3.2$ in Figure $6 \mathrm{~b}$, the phase relation between the waves measured by the two ground magnetometer stations used (with the cross phase technique: see (Waters et al., 1991)) was reversed from the normal relation, indicating that the $L$ value might be within the plasmapause (Menk et al., 1999). For this reason, we made the model plasmapause (Figure 2) bulge out slightly at MLT $\sim 10.3$ to put that data point near the model plasmapause (Figure 6b). Note that we have no data from EUV at this local time.

\subsection{Floor on the Electron Density at Large $L$}

Figure 3e shows that there is a large variation in the electron density at large $L$ in the region outside the plasmasphere. Values range from 1 to $20 \mathrm{~cm}^{-3}$ at the times indicated in Figure 3e. The EUV image shows a significant amount of light at large $L$ in the original EUV image, indicating that the density at large $L$ may be a significant fraction of $40 \mathrm{~cm}^{-3}$, the density typically associated with the EUV plasmasphere threshold (Goldstein et al., 2003). Because of this, we put a floor (lower limit) on $n_{e}$ near the upper limit of the 1 to $20 \mathrm{~cm}^{-3}$ range observed by RPI (Figure 3e), $(11 \pm 4 \cos (2 \pi(M L T-18) / 24)) \mathrm{cm}^{-3}$.

\subsection{Plasmapause Width}

From the EUV image (Figure 1a), it was apparent that the plasmapause was more narrow at dawn than at dusk. We chose to allow a sinoidal variation of the plasmapause width with respect to MLT. The maximum width is $0.4 R_{E}$ at MLT $=17$ hours, and the minimum width is $0.1 R_{E}$ at MLT $=5$ hours. Within that width, the equatorial density varies linearly from the value in the 
plasmasphere to the value outside the plasmasphere. (A median plasmapause width from Carpenter and Anderson (1992) is 0.1-0.2 $R_{E}$.)

\subsection{Polar Cap}

Outside of $L=13$ (based on the dipole field model), we assume that we are in the polar cap. (Our emphasis in this model is the plasmasphere rather than the polar cap. A more accurate description of this region would require a more accurate magnetic field model.) Within the polar cap, we model the electron density using the formula given by Gallagher et al. (2000),

$$
\log _{10}\left(n_{e}\right)=-3.09 \log _{10}(h)+13.5 \text {, }
$$

where $h$ is the altitude in $\mathrm{km}$ and $n_{e}$ is the density in $\mathrm{cm}-3$.

\subsection{Field Line Dependence}

For the field line density variation, we used the model of Denton et al. (2002) based on electron density measurements from the Polar spacecraft. Along the field line, a power law dependence is assumed with respect to the geocentric radius,

$$
n_{e}=n_{e 0}\left(L R_{E} / R\right)^{\alpha},
$$

where $n_{e 0}$ is the equatorial electron density. The value of $\alpha$ is given by

$$
\alpha=\alpha_{\text {model }}=8.0-3.0 \log _{10} n_{e 0}+0.28\left(\log _{10} n_{e 0}\right)^{2}-0.43 L .
$$

If this formula led to $\alpha<0$, we raised $\alpha$ to zero. Also, motivated partly by the results of Denton et al. (2004) (showing that the density at $R \sim 2 R_{E}$ often levels off at large $L$ to $n_{e} \sim 100 \mathrm{~cm}^{-3}$ ) and partly by the fact that low density at low altitude can cause numerical problems in simulations, we imposed an additional criterion on $\alpha$. If the density at a radius of $2 R_{E}$ based on Equation 6 with Equation 7 is less than $100 \mathrm{~cm}^{-3}, \alpha$ is increased so as to bring the density at $R=2 R_{E}$ up to $100 \mathrm{~cm}^{-3}$.

Calculations of the field line dependence of mass density using field line resonance mode harmonics (Takahashi et al., 2004) indicate that $\alpha$ might better be modelled with a low value $0-1$ throughout the (closed) magnetosphere. At this time, we do not know whether Equation 7 or $\alpha \sim 0-1$ better describes 


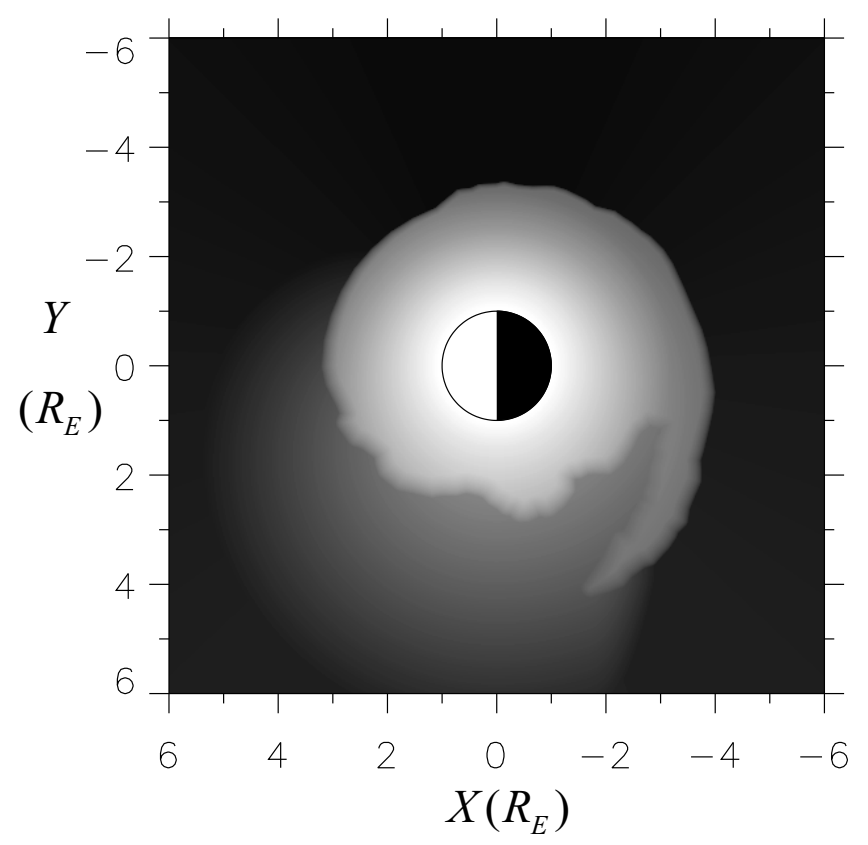

Fig. 7. Grayscale plot of the equatorial density as a function of SM coordinates $X$ and $Y$.

the field line difference. The difference in the simulated EUV image based on our model (Figure 1b using Equation 7) is not great.

\section{Discussion}

Our goal has been the generation of a mass density model that is realistic (accurate within perhaps a factor of 2) in the regions where we have good information about the density (plasmasphere proper and plasmatrough at dusk) and at least reasonable in the regions where our diagnostics are not so good (especially the outer plasmatrough). The first input to our model is the EUV image of resonantly scattered solar EUV photons at 29 August 20001519 UT (Section 2.1, Figure 1a). This image provides the plasmapause boundary (Section 2.2, Figure 2) and some evidence regarding the MLT dependence of density in the plasmasphere and density of the plume relative to the surrounding plasma (Section 2.4) and regarding the density in the plasmatrough at large $L$ (Section 2.6). The local electron density measurements from IMAGE RPI (Section 2.3, Figure 3) provide the electron density in the plasmasphere proper (plasmasphere excluding the plume) as a function of $L$ and some guidance concerning the electron density in the plasmatrough (Section 2.6). The inferred mass density values from the field line resonant mode provide the average ion mass (assumed to be constant throughout the magnetosphere) and the density in the dusk region outside the plasmasphere (Section 2.5, Figure 6). Figure 7 shows the logarithm of the model equatorial density (either electron 
or mass density, since the grayscale levels are uncalibrated) as a function of SM coordinates $X$ and $Y$.

From the equatorial density, we use the field line dependence model of Denton et al. (2002) to generate a three dimensional model of magnetospheric electron and mass density. Using the model electron density as an input, a simulated EUV image is generated. The procedure is as follows. Line of sight vectors are calculated over a 151 by 151 grid of $0.6^{\circ}$ pixels using IMAGE orbit and attitude data for the epoch (an EUV image is roughly $85^{\circ}$ in a direction normal to the scan plane of the spacecraft and $360^{\circ}$ in the transverse direction, and has pixels that are $0.6^{\circ}$ to a side), and then the pixel line of sight integrated $\mathrm{He}+30.4-\mathrm{nm}$ signal is estimated under the assumption of a constant ratio of $\mathrm{He}+$ to electrons. The numerical integration extends from the spacecraft out to $L=9$ on the far side of Earth, excluding any part subtended by the Earth's shadow, and is simplified by the fact that although the volume of any voxel (three dimensional pixel) along the line of sight scales as $r^{2}$, where $r$ is the distance to the voxel, the radiation collected by the sensor from that voxel falls as $r^{-2}$. The electron density within a voxel is taken to be the model electron density at its center. The simulation assumes a perfect detector and, up to a scale factor, should be consistent with EUV images that have been processed to remove geometric distortions and the effects of spacecraft rotation, and have been flat fielded and registered during ground processing.

The resulting image is shown in Figure 1b. Though presented last, the comparison between Figure 1a (real EUV image) and Figure 1b (simulated EUV image using our density model) provided additional input to the model. For instance, based on the results from the simulated image the width of the plume was decreased where there was some uncertainty about the exact location of the outer edge, and the ratio of density in the plume relative to the slot region just inside the plume was increased. The general similarity of Figure 1a and Figure 1b provides support for the overall model.

\section{Acknowlegements}

Work at Dartmouth was supported by NSF grant ATM-0245664 and NASA grant NAG5-11825. Work at UCLA was supported by NASA Graduate student research fellowship NASA NGT5-117. We thank the institutes who maintain the IMAGE magnetometer array. The authors thank the SAMNET team for providing the SAMNET and BGS magnetometer data. SAMNET is a PPARC National Facility operated by Lancaster University and BGS is a NERC funded facility. 


\section{References}

Bauer, S.J., and R.G. Stone, Satellite observations of radio noise in the magnetosphere, Nature, 218, 1145-1147, 1968.

Benson, R.F., P.A. Webb, J.L. Green, L. Garcia, and B.W. Reinisch, Magnetospheric electron densities inferred from upper-hybrid band emissions, Geophys. Res. Lett., submitted, 2004.

Berube, D., M. B. Moldwin, and J. M. Weygand, An automated method for the detection of field line resonance frequencies using ground magnetometer techniques, J. Geophys. Res., 108, NO. A9, 1348, doi:10.1029/2002JA009737, 2003.

Burch, J. L., Goldstein, J., and B. R. Sandel, Cause of plasmasphere corotation lag, Geophys. Res. Lett., 31, L05802, doi:10.1029/2003GL019164, 2004.

Carpenter, D.L, and R.R. Anderson, An ISEE/whistler model of equatorial electron density in the magnetosphere, J. Geophys. Res., 97, 1097, 1992.

Craven, P.D., D.L. Gallagher, and R.H. Comfort, Relative concentration of $\mathrm{He}+$ in the inner magnetosphere as observed by the DE 1 retarding ion mass spectrometer, J. Geophys. Res., 102 (A2), 2279-2289, 1997.

Dent, Z.C., I.R. Mann, F.W. Menk, J. Goldstein, C.R. Wilford, M.A. Clilverd, and L.G. Ozeke, A coordinated ground-based and IMAGE satellite study of quiet-time plasmaspheric density profiles, Geophys. Res. Lett., 30 (12), 1600, doi:10.1029/2003GRL016946, 2003.

Denton, R.E., and D.L. Gallagher, Determining the mass density along magnetic field lines from toroidal eigenfrequencies, J. Geophys. Res., 105, 27,717, 2000 .

Denton, R.E., D.-H. Lee, K. Takahashi, J. Goldstein, and R. Anderson, Quantitative test of the cavity resonance explanation of plasmaspheric Pi2 frequencies, J. Geophys. Res., 107, NO. A7, 10.1029/2001JA000272, 2002.

Denton, R.E., J. Goldstein, J.D. Menietti, and S.L. Young, Electron density in the magnetosphere, J. Geophys. Res., in press, 2004.

Gallagher, D. L., P. D. Craven, and R. H. Comfort, Global core plasma model, J. Geophys. Res., 105, 18,819, 2000.

Goldstein, J., M. Spasojevic, P.H. Reiff, B.R. Sandel, W.T. Forrester, D.L. Gallagher, and B.W. Reinisch, Identifying the plasmapause in IMAGE EUV data using IMAGE RPI in situ steep density gradients, J. Geophys. Res., 108, NO. A4, 1147, doi:10.1029/2002JA009475, 2003.

Luhr, H., A. Aylward, S.C. Bucher, A. Pajunpaa, K. Panjunpaa, T. Holmboe, and S.M. Zalewski, Westward moving dynamic substorm features observed with the IMAGE magnetometer network and other ground-based instruments, Ann. Geophys., 16 (4), 425-440, 1998.

Menk, F.W., D. Orr, M.A. Clilverd, A.J. Smith, C.L. Waters, D.K. Milling, and B.J. Fraser, Monitoring spatial and temporal variations in the dayside plasmasphere using geomagnetic field line resonances, J. Geophys. Res., 104, 19,955, 1999.

Reinisch, B.W. et al., The radio plasma imager investigation on the IMAGE 
spacecraft, Space Sci. Rev., 91, 319, 2000.

Sandel, B. R., R. A. King, W. T. Forrester, D. L. Gallager, A. L. Broadfoot, and C. C. Curtis, Initial results from the IMAGE extreme ultraviolet imager, Geophys. Res. Lett., 28, 1439, 2001.

Takahashi, K., R.E. Denton, R.R. Anderson, and W.J. Hughes, Frequencies of standing Alfvn wave harmonics and their implication for plasma mass distribution along geomagnetic field lines: Statistical analysis of CRRES data, J. Geophys. Res., in press, 2004.

Tobiska, W.K., T. Woods, F. Eparvier, R. Viereck, L. Floyd, D. Bouwer, G. Rottman, and O.R. White, The SOLAR2000 empirical solar irradiance model and forecast tool, J. Atmos. Sol.-Terr. Phys., 62 (14), 1233-1250, 2000 .

Tsyganenko, N.A., Modeling the Earth's magnetospheric magnetic-field confined within a realistic magnetopause, J. Geophys. Res., 100, 5599, 1995. Tobiska, W. K., Status of the SOLAR2000 solar irradiance model, Physics and Chemistry of the Earth, Part C: Solar, Terrestrial \& Planetary Science, Volume 25, Issues 5-6, Pages 383-386, 2000.

Waters, C.L., F.W. Menk, and B.J. Fraser, The Resonance Structure of Low Latitude Pc3 Geomagnetic-Pulsations, Geophys. Res. Lett., 18 (12), 22932296, 1991.

Wolf, R.A., Magnetospheric configuration, in Introduction to Space Physics, edited by M.G. Kivelson and C.T. Russell, pp. 288-329, Cambridge University Press, New York, NY, 1995.

Yeoman, T.K., D.K. Milling, and D. Orr, Pi2 Pulsation Polarization Patterns on the U-K Subauroral Magnetometer Network (Samnet), Planetary and Space Science, 38 (5), 589-602, 1990. 